\title{
Mechanomyography versus Electromyography, in monitoring the muscular fatigue
} Mihai T Tarata*1,2

\author{
Address: ${ }^{1}$ Department of Medical Informatics, University of Medicine and Pharmacy of Craiova, Bul. Antonescu 62, Craiova, Romania and \\ ${ }^{2}$ Corresponding address - 178 S Forker Bldg., Health \& Human Performance Dept., Iowa State University, Ames, Iowa, IA-50011, USA \\ Email: Mihai T Tarata* - mtarata@iastate.edu \\ * Corresponding author
}

Published: II February 2003

BioMedical Engineering OnLine 2003, 2:3

Received: 25 November 2002

Accepted: II February 2003

This article is available from: http://www.biomedical-engineering-online.com/content/2/I/3

(C) 2003 Tarata; licensee BioMed Central Ltd. This is an Open Access article: verbatim copying and redistribution of this article are permitted in all media for any purpose, provided this notice is preserved along with the article's original URL.

\begin{abstract}
Background: The use of the mechanomyogram (MMG) which detects muscular vibrations generated by fused individual fiber twitches has been refined. The study addresses a comparison of the MMG and surface electromyogram (SEMG) in monitoring muscle fatigue.

Methods: The SEMG and MMG were recorded simultaneously from the same territory of motor units in two muscles (Biceps, Brachioradialis) of the human $(n=18)$, during sustained contraction at $25 \%$ MVC (maximal voluntary contraction).

Results: The RMS (root mean square) of the SEMG and MMG increased with advancing fatigue; MF (median frequency) of the PSD (power density spectra) progressively decreased from the onset of the contraction. These findings (both muscles, all subjects), demonstrate both through the SEMG and MMG a central component of the fatigue. The MF regression slopes of MMG were closer to each other between men and women (Biceps 1.55\%; Brachialis 13.2\%) than were the SEMG MF slopes (Biceps 25.32\%; Brachialis 17.72\%), which shows a smaller inter-sex variability for the MMG vs. SEMG.

Conclusion: The study presents another quantitative comparison (MF, RMS) of MMG and SEMG, showing that MMG signal can be used for indication of the degree of muscle activation and for monitoring the muscle fatigue when the application of SEMG is not feasible (chronical implants, adverse environments contaminated by electrical noise).
\end{abstract}

\section{Background}

The force produced by a muscle under voluntary contraction is based on two mechanisms: the firing frequency and the recruitment of the motor units. The motor unit (MU) is a functional entity consisting of a motor neuron and the whole set of muscular fibers it innervates. Increasing either the firing frequency or the number of recruited MUs contributes to increasing the muscle force. The smoothness of the force output of a muscle is enhanced by the firing rate / recruitment interaction within the MU pool [1] and the mechanical filtering effect of the tissue, compen- sating for the discrete nature of the process, which builds up the actual muscle force from individual muscle fiber twitches. The variation of the firing rates of the motor units occurs simultaneously for all the motor units within a muscle, and even in different muscles acting on the same joint, according to the phenomenon of 'common drive' [2]. Accordingly, small variations of the output force acquired via a force transducer can be noticed. Also, the control loops involving Ia, Ib afferents are responsible for the occurrence of certain peaks within the output force in the 
isometric exercise, and others are due to the activation from higher levels [3].

Sustained muscular contractions externally associated with not being able to maintain a certain force lead to physiological fatigue, tremor or pain, localized in the specific muscle (localized muscular fatigue). Fatigue is defined as 'any reduction in the force generating capacity (measured by MVC) regardless of the task performed' [4]. The fatigued muscle develops tetanic tension more slowly than normal and prolongs the relaxation phase simultaneously with inner chemical changes. The failure in maintaining the motor task defines the endurance limit, and endurance time is the total duration of the task up to the endurance limit.

The myoelectric activity detected with surface electrodes (SEMG) may be considered as the summation of the electrical signals generated by a number of motor units, active within the same motor territory in the proximity of the electrodes. The SEMG signal is a convenient mean to study the muscle behavior under fatiguing exercise, as it proves time-dependent changes, provided care is taken to prevent cross talk from adjacent muscles.

Fatigue is associated with a compression of the PSD of the SEMG toward lower frequencies, from the very beginning of the voluntary contraction [5]. This is due to the reduction in the conduction velocity in direct relation with the muscular fiber membrane excitability and with neural adaptations, resulting in an increase of the lower frequency content of the signal. Fatigue is also associated with higher amplitudes of the SEMG signal toward the end of the exercise $[3,5,6]$. It has been shown that, in sustained motor tasks, changes at different levels, including motoneural discharge behaviour, develop before an endurance limit is reached, phenomenon called 'central fatigue' $[7,8]$. Central and peripheral fatigue develop together, and have to be seen not as a result, but as complementary elements of a complex strategy striving to insure the optimality of the motor behavior within the framework of available resources.

Under muscular contraction, mechanical vibrations occur, due to three main processes: (i) the inner muscular vibrations, which are the intrinsic components of the muscle contraction [9], (ii) oscillations of the human motor system, e.g. tremor and clonus [10], and (iii) artefacts. They are located in specific frequency ranges, with a certain overlapping: the artefacts - due to large movements show the lowest frequencies, possible tremor contributions are below $10 \mathrm{~Hz}$, in healthy subjects usually between 5.85 and $8.8 \mathrm{~Hz}[11]$, and the mechanical inner vibrations affect the range between 10 and $40 \mathrm{~Hz}$.

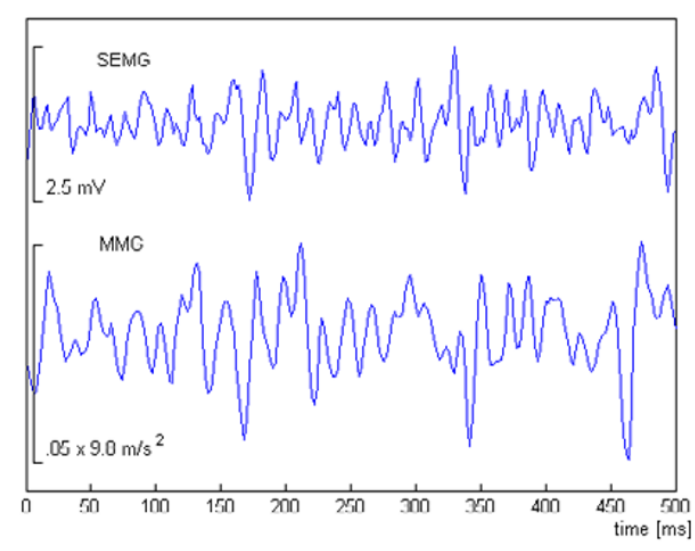

Figure I

Typical SEMG and MMG signals from the Biceps muscle (subject E). The figure shows SEMG (upper trace) and MMG (lower trace) signals, recorded from the same site of the Biceps muscle.

The MMG may be considered to reflect the mechanical muscle vibrations generated by the spatio-temporal summation of the individual muscle fiber twitches which are evoked through MU activation by the motor neurons. MMG recording is useful in experimental situations, when SEMG usage is not feasible, such as in complex working environments, and in areas with heavy electromagnetic pollution. The MMG was recorded with acceleration or sound transducers [10-14]. The technique of recording MMG was refined using piezoresistive silicon accelerometers in a surface mount package [15-17] to provide a reliable acquisition of MMG $[15,16]$. Preliminary results showed the potential of the MMG signal to monitor the muscle fatigue [15]. The EMG and MMG recorded simultaneously from the same muscle seem to have similar behavior $[3,15,16]$, i.e. the MF of SEMG and MMG decrease from the very beginning of the contraction, and their RMS values increase.

The actual study addresses another extensive comparison of the MMG and SEMG in monitoring the muscle fatigue, aiming to appreciate whether the MMG alone may be used to monitor the muscle behavior under fatiguing contraction. We are hypothesizing that MMG can indicate the degree of muscle activation and therefore can be used to monitor the development of the muscle fatigue, in the same way the SEMG has been proved to do.

\section{Methods}

Nine female, and nine male voluntary, healthy, motivated subjects participated with their consent, each group aged 
between 23 and 35. A pair of surface EMG self adhesive conductive gel electrodes $(22.5 \times 22.5 \mathrm{~mm}$ H59P, MVAP, USA), with their centres $25 \mathrm{~mm}$ apart from each other were placed on abrased, clean skin, longitudinally, immediately under the thickest point of the Biceps; a second similar pair of electrodes were placed on the Brachioradialis muscle of the same arm. The task involved a steady contraction, the subject sitting on a chair, with a $90^{\circ}$ anteflexion between the forearm and the arm, having to carry a weight hanging from the wrist via a soft belt. The shoulders were fixed with belts, to prevent involuntary rising movement during the exercise. The subject was asked to keep the forearm /arm angle at $90^{\circ}$ using the thumb to point to a marker located at the height of the forearm. Visual feedback was used to insure the position of the hand was stationary during the task; thus, given the constant resistant force, the contraction force was kept constant, on average. The $100 \% \mathrm{MVC}$ was estimated at first for the subject under test, as the maximum weight that the subject could sustain for two seconds, after a few short preliminary trials for learning and breaks between trials to avoid fatigue. Tests were performed for $25 \%$ MVC up to exhaustion, on different days. The level of contraction was chosen 25\% MVC for two reasons: (i) this is a normal level for the most regular tasks and (ii) to insure smaller mechanical oscillations, which are known to increase with the force of contraction. Two accelerometers (+-2 g, ICS Sensors, model 3031, USA) and author-made original amplifiers ( $\times 50000,10-250 \mathrm{~Hz}$ band pass filter, $250 \mathrm{~Hz}$ antialias filter) were used to pick up the mechanical vibrations of the two muscles. The accelerometers were placed between the SEMG electrodes to pick up the MMG on the axis orthogonal to the muscle from the same motor territory.

The SEMG signals were amplified $(\times 2000,100 \mathrm{M} \Omega$ input impedance, $100 \mathrm{~dB}$ CMRR, $250 \mathrm{~Hz}$ antialias filter, Beckman R611, USA) and acquired together with the MMG signals via a computerized acquisition system (DAP1200 Microstar Laboratories USA), at $500 \mathrm{~Hz}$ sampling rate on all the channels simultaneously.

The RMS, the PSD and the MF were computed for all the subjects from the original signals (SEMG, MMG) in successive buffers of $500 \mathrm{~ms}$, to ensure consistency across the data, according to the wide sense stationarity [17]. The power density spectra were computed via zero padding to 1024 point Fast Fourier Transform (FFT) for each data buffer, to allow the interpolation for a finer frequency resolution.

\section{Parameters}

PSD: The PSD of a stationary random process $x_{n}$ is estimated via the discrete-time Fourier transform:

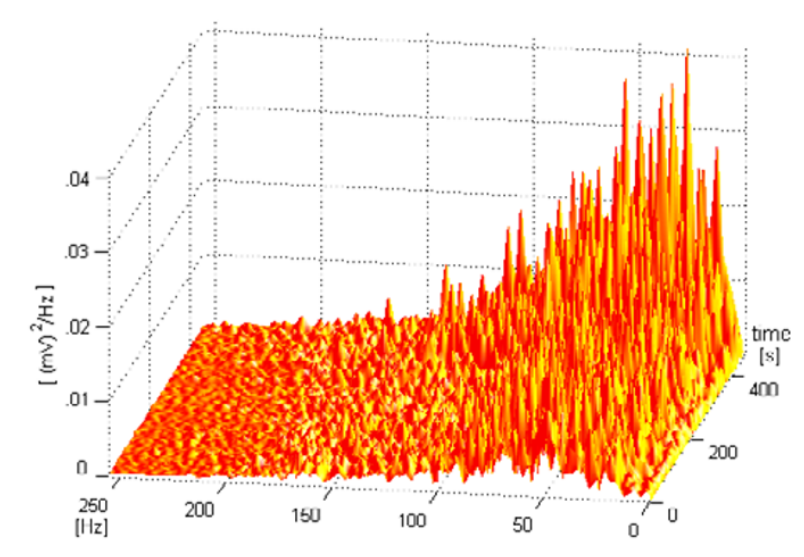

\section{Figure 2}

SEMG PSD evolution in time (subject E): Biceps muscle - surface plot. The figure shows the evolution in time of the Biceps muscle SEMG PSD. With increasing fatigue higher PSD peaks may be noticed.

where FFT is the Fourier transform of the specific sequence. Computed in this form, this estimation is called the periodogram. Because using windows other than the rectangular ones does not necessarily improve the estimate [18], a rectangular window has been used.

RMS: The RMS of the SEMG signal is considered the most reliable parameter in the time domain [19]. An increase of the SEMG RMS with advancing fatigue has been reported in many cases $[20,21]$. RMS is claimed to be not affected by the cancellation due to the motor unit action potential train superposition, which affects other processing techniques involving rectification [19]. It is also related to the power of the signal via the Parseval's relation [22], thus providing a direct connection with the frequency domain:

$R M S[s(t)]=\sqrt{\frac{\sum_{i=1}^{n} P S D_{i}}{n}}$

where $P S D_{i}-i^{\text {th }}$ PSD component

$n$ - the number of spectral components.

From (2) it follows that the overall average activity observed through the PSD of a certain SEMG segment, is expressed by the RMS value computed on the same specific SEMG segment of data.

$\operatorname{PSD}(\omega)=\left|F F T\left(x_{n}\right)\right|^{2}$ 


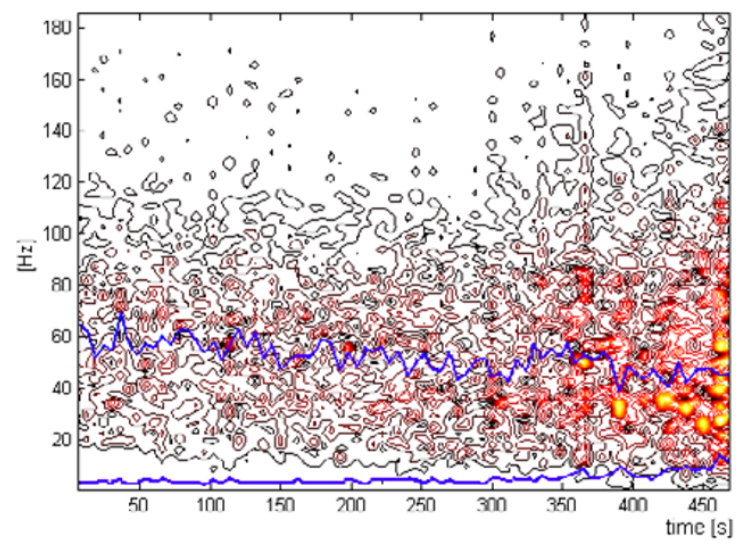

Figure 3

SEMG PSD evolution in time (subject E): Biceps muscle - contour plot. The evolution in time of the Biceps muscle SEMG PSD is shown in contour plot, computed to display at 15 levels the isolines of the matrix containing the succession of the spectra, from the beginning to the end of the contraction (black - zero level; red, yellow - higher PSD peaks). The PSD compression towards lower frequency and higher PSD peaks by the end of contraction, are noticeable. The middle trace (blue) shows the SEMG MF evolution; MF decreases with increasing fatigue, thus proving the PSD compression. The lower trace (blue) shows the SEMG RMS evolution; RMS increases with increasing fatigue.

The PSD and the RMS values were computed for all the signals (SEMG, MMG, both muscles) in all subjects. The evolution of the PSD with time is shown in contour plots, computed to display at 15 levels the isolines of the matrix containing the succession of the spectra, from the beginning to the end of the contraction.

MF: MF is the frequency value, separating the power spectrum into two equal surface regions. SEMG MF has been proved to be a reliable descriptor of the muscle fatigue evolution $[3,5,6,15,16]$ in the isometric exercise.

For all the subjects, the linear regression was performed on the RMS and MF evolutions to compute the slope (a1) and intercept point $(\mathrm{a} 0)$ parameters:

$M F_{e}=a 0_{M F}+a 1_{M F} \cdot t$

$R M S_{e}=a 0_{R M S}+a 1_{R M S} \cdot t$

where: $\mathrm{MF}_{e}$ - estimated MF, $\mathrm{RMS}_{\mathrm{e}}$ - estimated RMS; a0 intercept point; $\mathrm{a} 1$ - the slope; $\mathrm{t}$ - time [s].

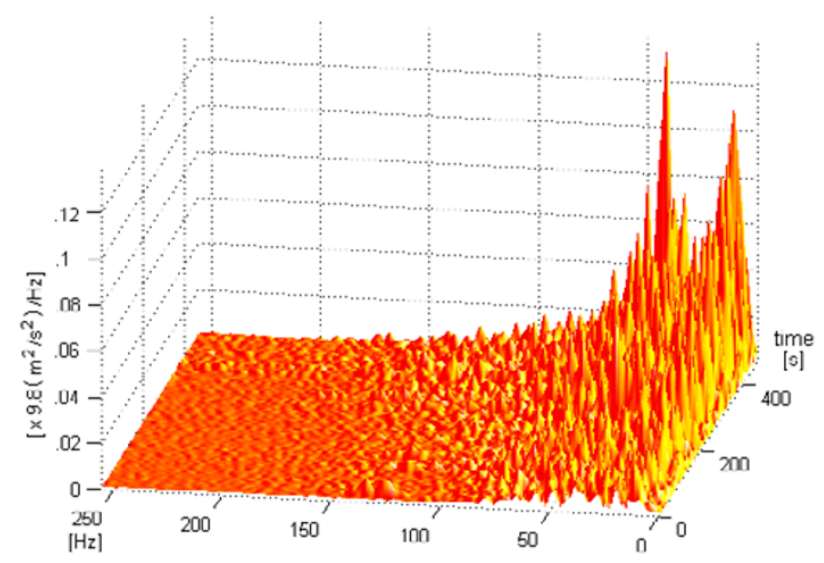

Figure 4

MMG PSD evolution in time (subject E): Biceps muscle. The figure shows the evolution in time of the Biceps muscle MMG PSD. With increasing fatigue higher PSD peaks may be noticed.

\section{Results}

The PSD spectra evolution computed on successive 500 ms signal segments from SEMG and MMG (typical example in Figure 1), show a compression toward lower frequencies with increasing fatigue both for the SEMG signal (Figure 2 - Biceps muscle) and the MMG signal (Figure 4 - Biceps muscle), starting at the very beginning of the contraction, in all cases. This can be also seen on the contour plots, both for the SEMG signal (Figure 3 - Biceps muscle) and the MMG signal (Figure 5 - Biceps muscle).

The MF evolution shows a progressive decrease from the very beginning of the task (Figure 6) in the both muscles and for the both signals, SEMG and MMG as well.

An increase in the RMS is seen with developing fatigue, both for the SEMG signal (Figure 7) and for the MMG signal (Figure 8).

The similar evolutions of both the SEMG and MMG, as above illustrated, support our hypothesis that MMG also indicates the degree of muscle activation and can be used to monitor the development of the muscle fatigue.

\section{Statistical analysis}

Tables 1 and 2 show the slopes and intercept points (average, standard deviation, min, $\max$ ) of the SEMG and MMG MF and RMS, for the two muscles in women, men and total. 


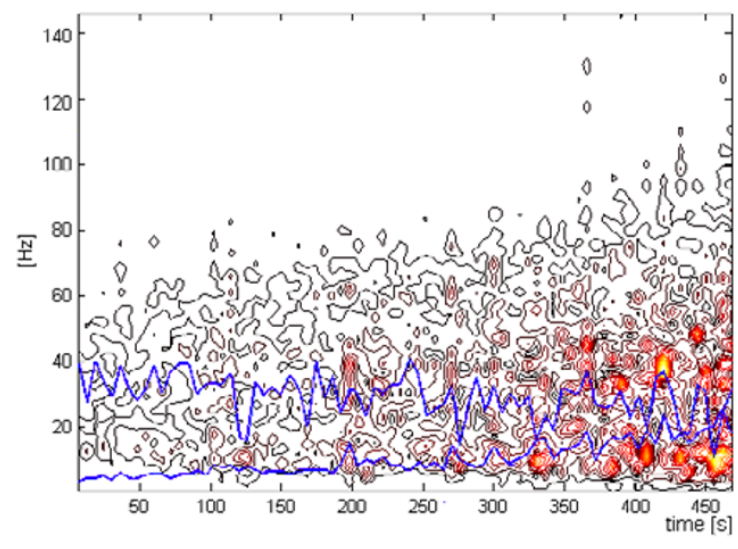

Figure 5

MMG PSD evolution in time (subject E): Biceps muscle. The evolution in time of the Biceps muscle MMG PSD is shown in contour plot, computed to display at 15 levels the isolines of the matrix containing the succession of the spectra, from the beginning to the end of the contraction (black zero level; red, yellow - higher PSD peaks). The PSD compression towards lower frequency and higher PSD peaks by the end of contraction, are noticeable. The middle trace (blue) shows the MMG MF evolution; MF decreases with increasing fatigue, thus proving the PSD compression. The lower trace shows the MMG RMS evolution; RMS increases with increasing fatigue.

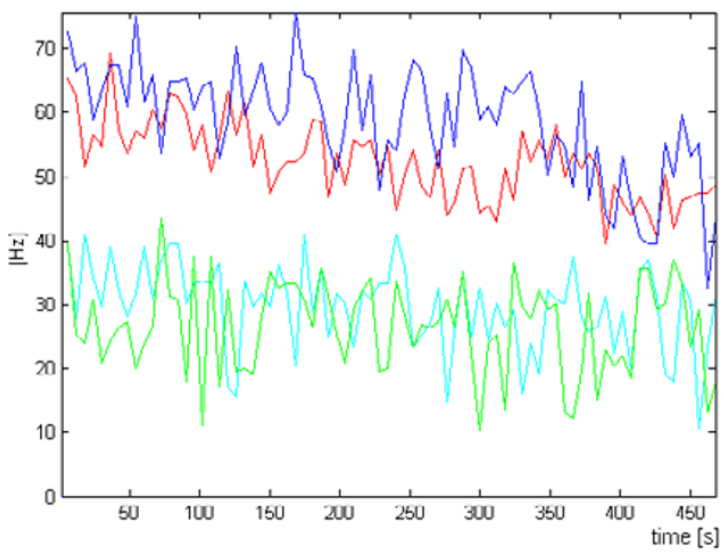

Figure 6

SEMG MF and MMG MF evolutions with increasing fatigue (subject E). MF evolutions are shown, both for the Biceps and Brachioradialis muscles (red - Biceps SEMG MF, blue - Brachioradialis SEMG MF, cian - Biceps MMG MF, green - Brachioradialis MMG MF). An overall decrease can be noticed starting at the very beginning of the contraction, which proves the central component of the fatigue.

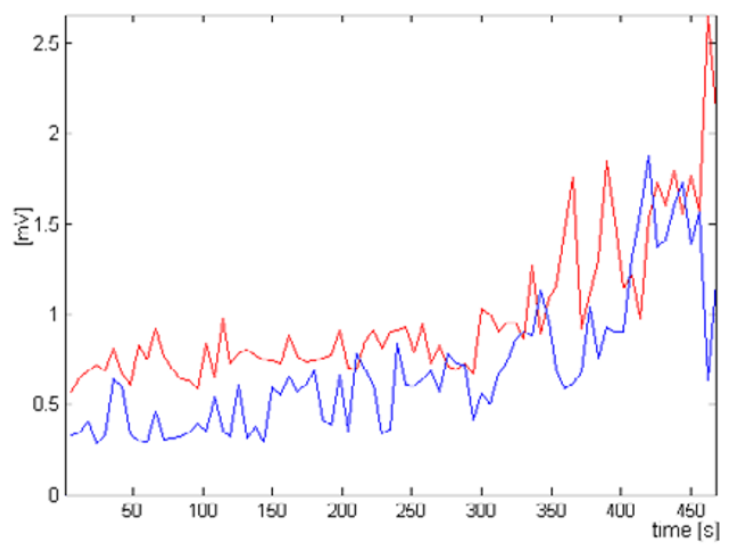

Figure 7

SEMG RMS evolutions with increasing fatigue (subject E). SEMG RMS evolutions are shown, both for the Biceps (red) and Brachioradialis (blue) muscles. An overall increase can be noticed from the very beginning of the contraction, more pronounced as time elapses.

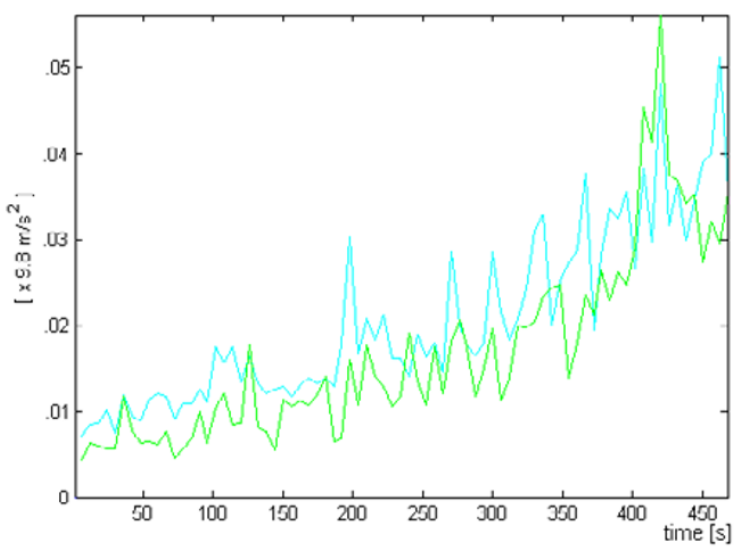

\section{Figure 8}

MMG RMS evolutions with increasing fatigue (subject E). MMG RMS evolutions are shown, both for the Biceps (cian) and Brachioradialis (green) muscles. An overall increase can be noticed from the very beginning of the contraction, more pronounced as time elapses. 
Table I: MF Slope (a I) and Intercept Point (a0)

\begin{tabular}{|c|c|c|c|c|c|c|c|c|c|}
\hline \multicolumn{2}{|c|}{ MF } & \multicolumn{2}{|c|}{ SEMG Bic. } & \multicolumn{2}{|c|}{ SEMG Brch. } & \multicolumn{2}{|c|}{ MMG Bic. } & \multicolumn{2}{|c|}{ MMG Brch. } \\
\hline & & al $[\mathrm{Hz} / \mathrm{s}]$ & $\mathrm{a} 0[\mathrm{~Hz}]$ & al $[\mathrm{Hz} / \mathrm{s}]$ & $\mathrm{a} 0[\mathrm{~Hz}]$ & al $[\mathrm{Hz} / \mathrm{s}]$ & $\mathrm{a} 0[\mathrm{~Hz}]$ & al $[\mathrm{Hz} / \mathrm{s}]$ & $\mathrm{a} 0[\mathrm{~Hz}]$ \\
\hline \multirow[t]{4}{*}{ Women } & Mean & -0.0358 & 59.1133 & -0.0299 & 57.3536 & -0.0187 & 27.6334 & -0.0091 & 25.1323 \\
\hline & Stdev. & 0.0130 & 10.1258 & 0.0231 & 7.2358 & 0.0113 & 5.0131 & 0.0054 & 3.0364 \\
\hline & Min. & -0.0390 & 46.0212 & -0.0573 & 50.1293 & -0.0520 & 20.1532 & -0.0185 & 19.2341 \\
\hline & Max. & -0.0157 & 83.5092 & -0.0091 & 64.5349 & -0.0023 & 32.9022 & -0.0033 & 26.1233 \\
\hline \multirow{4}{*}{ Men } & Mean & -0.0273 & 62.01 & -0.0310 & 62.9127 & -0.0193 & 30.2104 & -0.0097 & 27.7366 \\
\hline & Stdev. & 0.0145 & 7.1545 & 0.0199 & 6.3341 & 0.0092 & 5.0028 & 0.0071 & 3.2189 \\
\hline & Min. & -0.0573 & 52.687 I & -0.0552 & 51.2386 & -0.0456 & 25.2101 & -0.0433 & 23.3129 \\
\hline & Max. & -0.0123 & 82.5331 & -0.0019 & 69.4458 & 0.0012 & 37.3881 & 0.0113 & 29.9329 \\
\hline \multirow[t]{4}{*}{ Total } & Mean & -0.0302 & 59.9532 & -0.0285 & 60.2338 & -0.0184 & 29.3321 & -0.0087 & 26.3426 \\
\hline & Stdev. & 0.0213 & 8.8345 & 0.0223 & 6.5769 & 0.0178 & 6.2347 & 0.0073 & 3.0187 \\
\hline & Min. & -0.0573 & 46.0212 & -0.0573 & 50.1293 & -0.0520 & 20.1532 & -0.0433 & 19.2341 \\
\hline & Max. & -0.0123 & 83.5092 & -0.0019 & 69.4458 & 0.0012 & 37.3881 & 0.0113 & 29.9329 \\
\hline
\end{tabular}

Table 2: RMS Slope (a I) and Intercept Point (a0)

\begin{tabular}{|c|c|c|c|c|c|c|c|c|c|}
\hline \multicolumn{2}{|c|}{ RMS } & \multicolumn{2}{|c|}{ SEMG Bic. } & \multicolumn{2}{|c|}{ SEMG Brch. } & \multicolumn{2}{|c|}{ MMG Bic. } & \multicolumn{2}{|c|}{ MMG Brch. } \\
\hline & & $\mathrm{al}[\mathrm{mV} / \mathrm{s}]$ & $\mathrm{a} 0[\mathrm{mV}]$ & $\mathrm{al}[\mathrm{mV} / \mathrm{s}]$ & $\mathrm{a} 0[\mathrm{mV}]$ & $\begin{array}{l}\text { al }[9.8 \\
\left.\mathrm{mm} / \mathrm{s}^{3}\right]\end{array}$ & $\begin{array}{l}\mathrm{a} 0[9.8 \\
\left.\mathrm{mm} / \mathrm{s}^{2}\right]\end{array}$ & $\begin{array}{l}\text { al }[9.8 \\
\left.\mathrm{mm} / \mathrm{s}^{3}\right]\end{array}$ & $\begin{array}{l}\mathrm{a} 0[9.8 \\
\left.\mathrm{mm} / \mathrm{s}^{2}\right]\end{array}$ \\
\hline \multirow[t]{4}{*}{ Women } & Mean & .0014 & .3563 & .0008 & .2428 & .0294 & 6.6649 & .0943 & 9.6030 \\
\hline & Stdev. & .0008 & .1182 & .0004 & .1360 & .0135 & 2.3175 & .0547 & 3.8394 \\
\hline & Min. & .0004 & .1027 & .0002 & .0316 & .0165 & 3.171 & .0375 & 1.5495 \\
\hline & Max. & .0022 & .4735 & .0013 & .4459 & .054 & 9.828 & .189 & 13.1565 \\
\hline \multirow[t]{4}{*}{ Men } & Mean & .0029 & .6028 & .0019 & .4653 & .06700 & 9.3243 & .1103 & 9.1936 \\
\hline & Stdev. & .0025 & .2908 & .0014 & .2921 & .0462 & 6.4352 & .0472 & 6.1840 \\
\hline & Min. & .0002 & .3378 & .0002 & .1636 & .0135 & .3015 & .0465 & 1.5000 \\
\hline & Max. & .0065 & 1.2376 & .0040 & .9496 & .1710 & 21.8565 & .1935 & 19.9455 \\
\hline \multirow[t]{4}{*}{ Total } & Mean & .0021 & .4950 & .0014 & .3679 & .0505 & 8.1608 & .1033 & 9.3727 \\
\hline & Stdev. & .0022 & .2582 & .0012 & .2568 & .0398 & 5.1008 & .0495 & 5.1318 \\
\hline & Min. & -0.0002 & .1027 & .0002 & .0316 & .0135 & .3015 & .0375 & 1.5000 \\
\hline & Max. & .0065 & 1.2376 & .0040 & .9496 & .1710 & 21.8565 & .1935 & 19.9455 \\
\hline
\end{tabular}

The data (Tables 1,2 ) are graphically illustrated in figures $9,10,11,12,13,14$. Statistical comparisons using T-test (two-sample assuming unequal variances) were performed (significance level: 0.05). The null hypothesis (H0) assumed the equality of the compared parameters (mean values).

In all cases (Figure 9 - SEMG and MMG, both muscles) the average slope (a1) of the MF evolution is negative. The MMG MF regression slopes are closer to each other between men and women (Biceps 1.55\%; Brachioradialis $13.2 \%$ ) than are the SEMG MF slopes (Biceps 25.32\%; Brachioradialis $17.72 \%$ ); this shows a smaller inter-sex variability for the MMG vs. SEMG.
In all cases (Figure 11 - SEMG, Figure $13-M M G$, both muscles) the average slope (a1) of the RMS evolution is positive. The analysis of the RMS shows comparable slopes (a1) in women and men for the SEMG signal (Figure 11) between the two muscles, while for the MMG (Figure 13), they are comparable in men between the Biceps and Brachioradialis, but different in women.

\section{Discussion}

The local variations in the MF and the RMS are due to small local variations in the activation of the muscle. That is why areas of higher activation strips can be noticed in the contour plots (Figures 3,5). The mechanical resultant of the reinforcements in the activation (better illustrated by the RMS peaks) leads to oscillations of the output force 


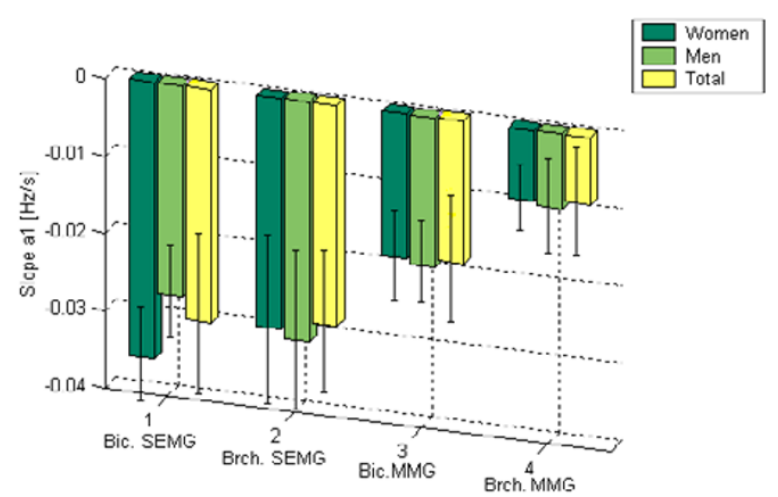

Figure 9

Mean Slope (a I) of MF Evolution. The MF evolution mean slope (al) is shown for the both muscles (Biceps \& Brachioradialis) and for the both signals (SEMG and MMG) for the groups of Women, Men and Total (see legend). In all the cases the MF slope is negative (MF decreases in time), showing the compression of PSD toward lower frequencies. The standard deviations are shown.

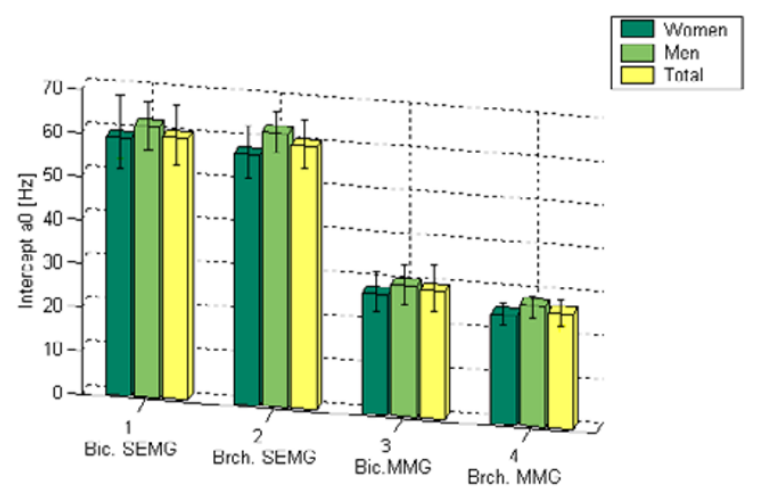

\section{Figure 10}

Mean Intercept Point (a0) of MF Evolution. The MF evolution mean intercept point $(\mathrm{a} 0)$ is shown for the both muscles (Biceps \& Brachioradialis) and for the both signals (SEMG and MMG) for the groups of Women, Men and Total (see legend). It is comparable between men and women in all cases, for the SEMG and MMG. The standard deviations are shown.

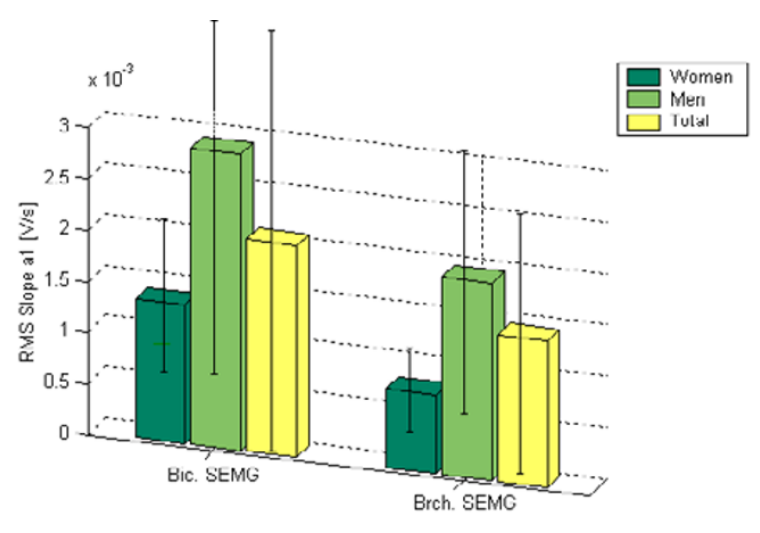

\section{Figure I I}

Mean Slope (a l) of SEMG RMS Evolution. The SEMG RMS evolution mean slope (al) is shown for the both muscles (Biceps \& Brachioradialis) for the groups of Women, Men and Total (see legend). The standard deviations are shown.

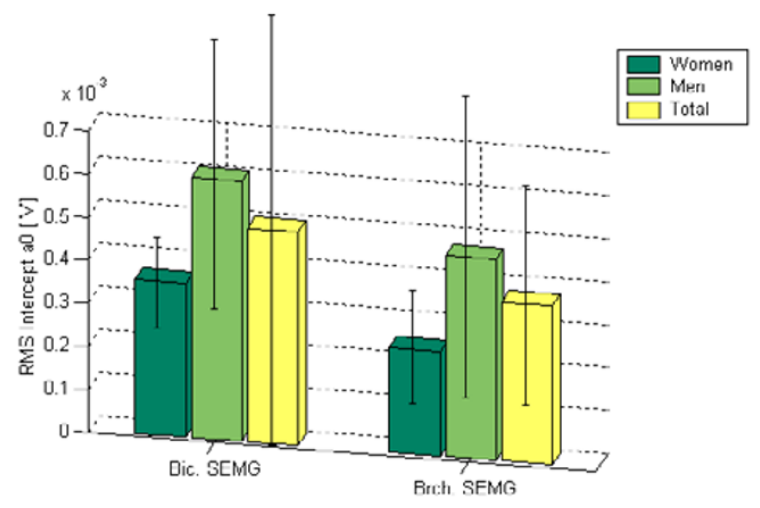

Figure 12

Mean Intercept Point (a0) of SEMG RMS Evolution. The SEMG RMS evolution mean intercept point $(\mathrm{a} 0)$ is shown for the both muscles (Biceps \& Brachioradialis) and for the groups of Women, Men and Total (see legend). The standard deviations are shown. 


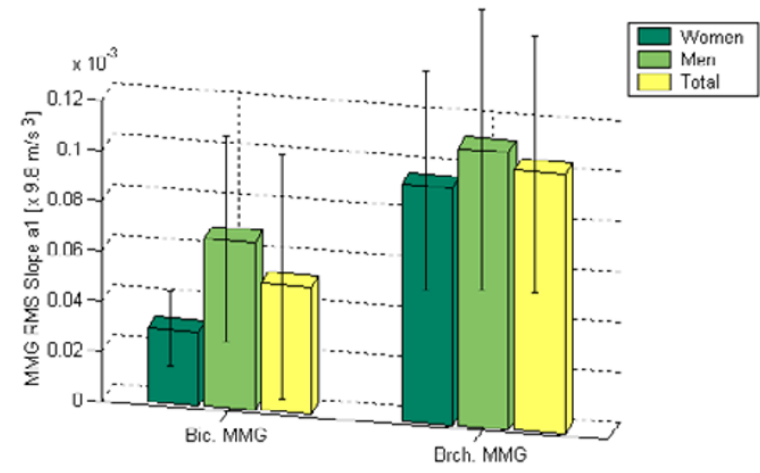

Figure I 3

Mean Slope (al) of MMG RMS Evolution. The MMG RMS evolution mean slope (al) is shown for the both muscles (Biceps \& Brachioradialis) for the groups of Women, Men and Total (see legend). The standard deviations are shown.

around its average. Meanwhile there is a progressive spreading up of the higher frequency activity within the PSD. This may be generated by progressive recruitment of new motor units in the both muscles. A larger frequency range toward the end of the task may also witness an increased activity of the controlling neural loops and modulating subsystems, as fatigue develops.

The amplitude of the MMG RMS progressively increases (Figure 8) with developing fatigue. This reflects increased twitch potentiation. An increase in the activation, possibly due to progressively higher number of active motor units together with a more synchronous firing may also play a role in the RMS increase. The same increase is shown by the SEMG (Figures 3, 7); this demonstrates that not only peripheral mechanisms are responsible for the MMG RMS increase.

MF decreases in all cases (both SEMG and MMG, both muscles, all subjects) from the very beginning of the muscle contraction. This proves the central intervention in modulating the activation with increasing fatigue (central fatigue). The MF decrease may be the effect of the centrally generated progressive alteration of the activation of individual MNs as the relaxation time increases; this may be explained by the withdrawal of the tonic fusimotor driven spindle-support via the fusimotor loop [23]. As the muscle afferents provide up to $30 \%$ excitation to the $\mathrm{MN}$, it seems that this mechanism stays as an important provision of intervention. The presynaptic inhibition,

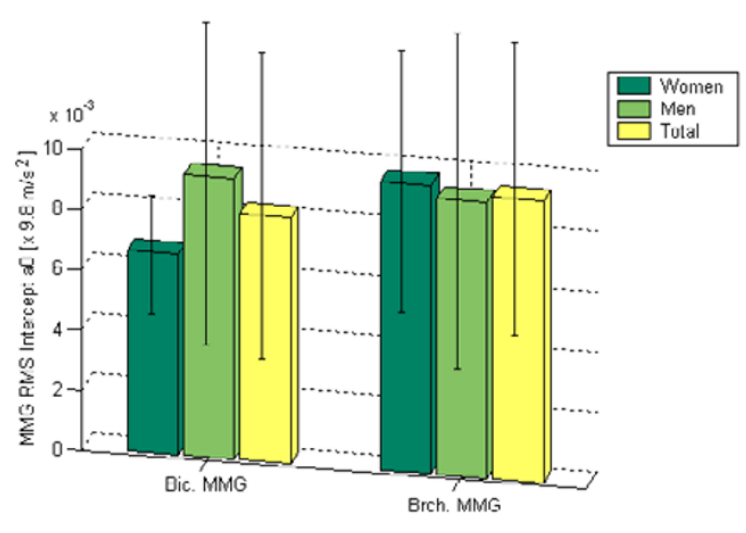

Figure 14

Mean Intercept Point (a0) of MMG RMS Evolution. The MMG RMS evolution mean intercept point (a0) is shown for the both muscles (Biceps \& Brachioradialis) and for the groups of Women, Men and Total (see legend). The standard deviations are shown.

may also contribute to reducing the fusimotor support with advancing fatigue and affects the Ia and Ib afferents. More, Ib inhibitory postsynaptic potentials in MNs also decline in sustained muscular contractions, and the gain of force feedback loop is reduced. The group II non-spindle muscle afferents have increased discharge rates with increasing fatigue, also contributing to the inhibitory effect. Meanwhile, the alpha MNs are centrally inhibited by group III and IV muscle afferents, activated by the accumulation of metabolites in the fatiguing muscle, but this is expected to come into action later than from the beginning, to further accelerate the process. This may be witnessed by the nonlinear spreading up of the frequency range and increase in RMS.

The above discussion shows the intimate link between the EMG and the MMG as functionally related signals; they both witness the neural activation of the muscular fibers and its mechanical effect, respectively. This functional link helps explaining the difference between the MF intercept points in SEMG and MMG (Figure 10). The MMG itself results as a spatio-temporal fused effect of the muscle fiber twitches. Hence, the MMG frequency spectrum is located lower than the SEMG spectrum on the frequency scale. This effect is further enhanced by the filtering effect of the tissue between the contraction site and the transducer. This mechanical filtering effect may also explain smaller inter-sex variability shown by the MF slope for the MMG compared to the SEMG. 
The MMG MF slopes (Figure 9) are statistically different between the Biceps and Brachioradialis muscles in women, men and total. In the isometric contraction, addressed by this work, this muscle shows a decrease of the MF with fatigue, less accentuated than the Biceps muscle shows. Even if the both muscles (Biceps and Brachioradialis) are intensively worked out, they are differently loaded, relative to their individual MVCs. This may influence the balance between the centrally modulated activation and the peripheral phenomena in each of them and may explain the difference. The above explanation may also address the significant difference in MMG RMS slopes (Figure 13) between Biceps and Brachioradialis muscles.

A study [24] brought results in agreement with our results regarding the evolution of the PSD and RMS. The study [24] mentions that the SEMG and MMG give information on the localized fatigue. Our results show that both the SEMG and MMG demonstrate the central component of the fatigue (the decrease in MF from the very beginning of the contraction). That is why the MMG is as valuable as SEMG in exploring not only peripheral fatigue, but also sensorimotor interaction and central intervention in the development of the muscular fatigue.

\section{Conclusion}

This study addresses a quantitative comparison (MF, RMS) of accelerometer-based MMG and SEMG. It brings additional evidence on the central fatigue and provides means of quantifying the fatigue development in the isometric exercise.

According to this study, the MMG signal can be used for indication of the degree of muscle activation and for monitoring the muscle fatigue development in the isometric contraction.

The study also advocates the use of the integrated accelerometer-based MMG acquisition. An accelerometer-based MMG transducer is selective, not dependent of the environmental acoustical and electrical noise and it also provides positional information on motor-unit territory. The transducer can be incorporated into a miniature, stand-alone, radio-frequency-powered device, which can be chronically implanted in the muscle. As such, it has multi-faceted potential for future research in movement neuroscience in adverse environments, when it is not feasible to use the SEMG: complex working environments, and situations wherein the SEMG signal is contaminated by electrical noise.

The work also encourages further research on the use of MMG in dynamic contraction.

\section{Authors' contributions}

MTT conceived the study, conceived and developed the macro-model for the MMG transducer, conceived and performed the experiments, performed the data analysis, drafted the manuscript.

\section{References}

I. De Luca CJ Control properties of motor units. J Exp Biol 1985, I | 5:125-136

2. De Luca CJ Control Scheme Governing concurrently Active Human Motor Units During Voluntary Contractions. Journal of Physiology 1982, 329: I 29-142

3. Tarata MT Sensorimotor interactions within the context of muscle fatigue. In: "Sensorimotor Control" (Dengler R., Kossev A., eds.), NATO Science Series, Series I: Life and Behavioural Sciences 200I, 326:84-91

4. Bigland-Ritchie $B$ and Woods JJ Changes in muscle contractile properties and neural control during human muscular fatigue. Muscle \& Nerve 1984, 7:691-699

5. Stulen FB and De Luca CJ Frequency parameters of the myoelectric signal as a measure of muscle conduction velocity. IEEE Trans Biomed Eng 198I, BME-28:515-522

6. Tarata M Monitoring the Evolution of the Muscular Fatigue, Via New Parameters developed from the SEMG Signal. In Proceedings of the ECSAP'97 The First European Conference on Signal Analysis and Prediction: 1997 June 24-27; Prague. ICT Press ISBN 807080-282-0; 1997, 431-434

7. Bigland-Ritchie $B$ Central and peripheral fatigue in sustained maximum voluntary contractions of human quadriceps muscle. Clinical Science and Molecular Medicine 1978, 54:609-6I4

8. Gandevia SC Central fatigue: critical issues, quantification and practical implications. Adv Exp Med Biol 1995, 384:28I-294

9. Barry DT Vibrations and sounds from evoked muscle twitches. Electromyogr \& Clin Neurophysiol 1992, 32:35-40

10. laizzo PA and Pozos RS Analysis of multiple SEMG and acceleration signals of various record lengths as a means to study pathological and physiological oscillations. Electromyogr \& Clin Neurophysiol 1992, 32:359-367

II. Comby B, Chevalier G and Bouchoucha M A new method for the measurement of tremor at rest. Archives Internationales de Physiologie, de Biochimie et de Biophysique 1992, 100:73-78

12. Watanabe $M$, Itoh $\mathrm{Y}, \mathrm{Mita} \mathrm{K}$ and Akataki $\mathrm{K}$ Technical aspects of mechanomyography recording with piezoelectric contact sensor. Med Biol Eng Comput 1998, 36(5):557-56I

13. Watanabe M, Mita K, Akataki $\mathrm{K}$ and Itoh $\mathrm{Y}$ Mechanical behaviour of condenser microphone in mechanomyography. Med Biol Eng Comput 2001, 39(2): 195-201

14. Hemmerling TM Phonomyography - Acoustic myography using condenser microphones. Anaesthesia and intensive care/Australian society of 2002, 30(4):532-533

15. Tarata MT, Spaepen A and Puers R A new technique for measuring muscle activity: the accelerometer MMG. In Abstracts, Higher Nervous Control of Posture and Locomotion: Parallel and centralized Control Mechanism: 200I March 19-2 I; Okazaki, Japan (Edited by: Mori $S$, Stuart DG, Wiesendanger M) National Center for Physiological Sciences 2001, I32-133

16. Tarata MT, Spaepen A and Puers R The Accelerometer MMG Measurement Approach in Monitoring the Muscular Fatigue. MEASUREMENT SCIENCEREVIEW, ISSN I335-887I 200I, I:47-50[http://www.measurement.sk]

17. Tarata MT Electronica medicala. ISBN.973-8025-42-7, Craiova, SITECH 1999 ,

18. Merletti $\mathrm{R}$ and Lo Conte LR Advances in processing of surface myoelectric signals - Part I. Medical \& Biological Engineering \& Computing 1995, 5:361-372

19. Basmajian JV and DeLuca CJ Muscles Alive. Their functions revealed by Electromyography. Baltimore, William \& Wilkins 1985,

20. Krogh-Lund $C$ and Jorgensen $K$ Myo-electric fatigue manifestations revisited: power spectrum, conduction velocity, and amplitude of human elbow flexor muscles during isolated and repetitive endurance contractions at $30 \%$ maximal voluntary contraction. European Journal of Applied Physiology 1993, 66(2): $161-173$ 
21. Ohashi J Changes in relations between SSEMG and fatigue level by repeating fatiguing static contractions. Annals of Physiological Anthropology 1993, I 2(5):285-296

22. Rabiner RL and Gold $B$ Theory and Application of Digital Signal Processing. ISBN 0-13-9/4101. Englewood Cliffs, Prentice-Hall I975,

23. Hagbarth KE and Macefield VG The fusimotor system. Its role in fatigue. In: "Fatigue" (Edited by: Gandevia SC) Plenum Press, New York 1995, 259-270

24. Pascal M, Jorgensen LV, Sogaard K, L Arendt-Nielsen and Sjogaard G Development of muscle fatigue as assessed by electromyography and mechanomyography during continuous and intermittent low-force contractions: effects of the feedback mode. Eur J Appl Physiol 2002, 87(I):28-37

Publish with Bio Med Central and every scientist can read your work free of charge

"BioMed Central will be the most significant development for disseminating the results of biomedical research in our lifetime. "

Sir Paul Nurse, Cancer Research UK

Your research papers will be:

- available free of charge to the entire biomedical community

- peer reviewed and published immediately upon acceptance

- cited in PubMed and archived on PubMed Central

- yours - you keep the copyright 